\title{
Discussing Professor Yin Kejing's Drug Use Law for Mammary Hyperplasia Based on Data Mining Technology
}

\author{
Weiwei Xu, Wenjing Hu, Kejing Yin, Xu Du* \\ Shaanxi University of Traditional Chinese Medicine, Xianyang, 712046, Shanxi Province, China.
}

How to cite this paper: Weiwei $\mathrm{Xu}$, Wenjing Hu, Kejing Yin, Xu Du. (2021) Discussing Professor Yin Kejing's Drug Use Law for Mammary Hyperplasia Based on Data Mining Technology. International Journal of Clinical and Experimental Medicine Research, 5(3), 395-402.

DOI: 10.26855/ijcemr.2021.07.026

Received: June 11, 2021

Accepted: July 8, 2021

Published: July 29, 2021

*Corresponding author: $\mathrm{Xu} \mathrm{Du}$, Shaanxi University of Traditional Chinese Medicine, Xianyang, 712046, Shanxi Province, China.

Email: dxdmzhnsh@sina.com

\begin{abstract}
Objective: To explore the drug use characteristics and rules of Professor Yin Kejing in the treatment of mammary hyperplasia. Methods: Select the clinical prescription of traditional Chinese medicine for mammary hyperplasia in the past three months, establish a prescription database for traditional Chinese medicine treatment mastosis by using Excel software, use the relevant rule method and cluster analysis in data mining technology, and summarize the characteristics and rules of prescription drugs for traditional Chinese medicine. Results: A total of 80 prescriptions of traditional Chinese medicine were included, involving 170 flavors of traditional Chinese medicine, and the total frequency of use of traditional Chinese medicine was 1,784 times. Among them, the most frequently used traditional Chinese medicine, meridian, four qi and five flavors were Huangqi (80 times), foot yin liver meridian (1,040), temperature (825 times) and bitterness (938 times). The correlation analysis results show that the most supportive combination of traditional Chinese medicine is tulips and plum blossoms. The cluster analysis results show that there are three effective cluster groups. It can be concluded that the drug used to treat mammary hyperplasia is mainly qi-activating blood, supplements qi and blood, treats both specimens, and qi and removes blood stasis. Clinically, the combination of Huangqi-Peonyeoniae-leaked reed-Rebuilding, white mustard-Yanhusuo, and tulips-Zlum flower can be used, and can be dialectically treated according to different clinical classifications.
\end{abstract}

\section{Keywords}

Mammary Hyperplasia, Traditional Chinese Medicine Prescription, Data Mining, Drug Use Law, Professor Yin Kejing

Mammary gland hyperplasia (MCH) belongs to the category of the motherland's medicine "lact fet", "mammary combination" and "milk rhyffian" [1], which mostly manifests as periodic breast pain and often interacts with moods such as irritability, anxiety and irritability. It is a woman. The most common breast disease has the highest incidence of mammary diseases. Breast hyperplasia is a benign hyperplasia of mammary tissue that is neither inflammation nor tumor. Its clinical characteristics are unilateral or bilateral breast pain and the emergence of lumps. The disease occurs in young and middle-aged women aged 25-45, and its incidence accounts for 75\% of mammography, which is the most common breast disease in clinic [2]. In recent years, the incidence of the disease has increased year by year, and it is also becoming younger and younger [3]. There is a certain tendency to cancer in this disease, and its risk of malignant change is 2-4 times higher than that of normal women [4], so you should be vigilant about this disease, diagnose it early, and treat it early [5]. Professor Yin Kejing is a famous national traditional 
Chinese medicine practitioner. He has had a unique view of mammary hyperplasia for more than 50 years. Professor Yin believes that the occurrence of milk practism is closely related to the functions of the liver, spleen, kidneys, punching and other organs and meridians. From decades of clinical experience of medical treatment, he has summarized that "letting is the basis of loss of duty, liver qi is depressed, and blood stasis and phlegm". The construction of traditional Chinese medicine classification theory as standard. Professor Yin Kejing's clinical combination of modern pharmacological research and drug meridian, four qi, Wuwei and other treatments have significant therapeutic effects on mammary hyperplasia.

The author is lucky to follow Professor Yin Kejing's clinical study and collect Professor Yin Kejing's prescriptions prescriptions for the treatment of milk addiction. He will use the calculation of traditional Chinese medicine and drug frequency, frequency calculation and systematic clustering method to mine the data of Professor Yin Kejing's prescription for the treatment of mammary hyperplasia, analyze and explore its drug use laws and characteristics, and provide reference for clinical treatment of mammary hyperplasia.

\section{Data and methods}

\subsection{Data sources}

There were 80 cases in this study, all of them came from Yin kejing studio of the Second Affiliated Hospital of Shaanxi University of traditional Chinese medicine and Department of mammary gland of our hospital from October 2020 to December 2020. The excel table was established, and the prescription number, medication, four Qi, five flavor and meridian tropism were input into Microsoft Office Excel 2010 software to establish the prescription database of acupuncture and moxibustion for the treatment of hyperplasia of mammary glands.

\subsection{Statistical analysis of data}

Excel and access were used to establish the database, SPSS 19.0 was used for frequency analysis, and veka3.6.9 was used for association rule analysis. The comprehensive and systematic analysis of all the entered prescriptions was carried out to mine the association between the data and the relationship between the rules.

\subsection{Prescription entry check}

The name of traditional Chinese medicine is Chinese Pharmacopoeia (2015 Edition), and the category of traditional Chinese medicine refers to traditional Chinese medicine (6) and practical manual of traditional Chinese medicine (7) in national pharmacopoeia.

\subsection{Diagnostic criteria}

According to the criteria for diagnosis, syndrome differentiation and curative effect of breast augmentation disease approved by breast disease professional committee of Chinese society of traditional Chinese medicine surgery in 2015.

(1) Breast pain, tingling or dull pain, can radiate to the armpit, shoulder back, related to menstruation, mood, continuous 3 months or intermittent 3-6 months do not alleviate.

(2) Breast lumps, tenderness, increase and decrease with mood and menstrual cycle.

(3) The diagnosis was confirmed by X-ray mammography and/or B-ultrasound.

\subsection{Case inclusion criteria}

(1) 18-55 years old female patients with MGH who met the diagnostic criteria of MGH;

(2) Patients with normal heart, liver, kidney and hematopoiesis and regular menstrual cycle;

(3) No internal or external treatment for hyperplasia of mammary glands in the past 2 months.

\subsection{Exclusion criteria}

(1) Patients under 18 years old, over 55 years old, or during pregnancy or lactation;

Patients with life-threatening primary diseases such as cardiovascular, cerebrovascular, liver, kidney and hematopoietic system, mental diseases and Alzheimer's disease.

\section{Results}

\subsection{Statistics of Medication Frequency}

The frequency of drugs in Professor Yin Kejing's prescription for the treatment of hyperplasia of mammary glands was counted. The total frequency of drug use was 1,784 times. A total of 170 traditional Chinese medicines 
and 20 core traditional Chinese medicines were used. According to the analysis of the use frequency of 80 prescriptions, Astragalus membranaceus, Radix Paeoniae Alba, Rhizoma Chuanxiong, Angelica sinensis and Rehmannia glutinosa were the top five herbs. The results are shown in Table 1.

Table 1. Frequency analysis of the first 20 Chinese herbal medicines

\begin{tabular}{cccccc}
\hline Medicine & Frequency & Frequency & Medicine & Frequency & Frequency \\
\hline Astragalus membranaceus & 80 & $4.48 \%$ & Corydalis yanhusuor & 44 & $2.47 \%$ \\
Radix Paeoniae Alba & 77 & $4.32 \%$ & Trichosanthes kirilowii & 43 & $2.41 \%$ \\
rhizome of chuanxiong & 75 & $4.20 \%$ & Zedoary turmeric & 41 & $2.30 \%$ \\
Chinses angelica & 75 & $4.20 \%$ & Chinese atractylodes & 40 & $2.24 \%$ \\
Rehmannia glutinosa & 75 & $4.20 \%$ & Poria cocos & 31 & $1.74 \%$ \\
Rhaponticum uniflorum & 62 & $3.48 \%$ & Acorus tatarinowii & 31 & $1.74 \%$ \\
White mustard seed & 54 & $3.14 \%$ & Bergamot & 28 & $1.57 \%$ \\
\hline
\end{tabular}

\subsection{Analysis of four Qi, five flavors and meridian tropism}

The main meridians of drugs in the prescription were liver meridians, spleen meridians and heart meridians (see Table 2); The four Qi of drugs are mainly warm and cold (see Table 3); The five flavors of the drug are mainly bitter, sweet and pungent (see Table 4).

Table 2. Analysis of 80 prescription drugs

\begin{tabular}{ccc}
\hline Meridian tropism & Frequency & Frequency \\
\hline Liver & 1,040 & $22.80 \%$ \\
Spleen & 789 & $17.30 \%$ \\
Heart & 628 & $13.77 \%$ \\
Lung & 565 & $12.39 \%$ \\
Stomach & 548 & $12.01 \%$ \\
Kidney & 354 & $7.76 \%$ \\
Gallbladder & 188 & $4.12 \%$ \\
Large intestine & 178 & $3.90 \%$ \\
Pericardium & 108 & $2.37 \%$ \\
Bladder & 81 & $1.78 \%$ \\
Small intestine & 64 & $1.40 \%$ \\
Sanjiao & 18 & $0.39 \%$ \\
\hline
\end{tabular}

Table 3. Analysis of four Qi in 80 cases of prescription drugs

\begin{tabular}{ccc}
\hline Four Qi & Frequency & Frequency \\
\hline Temperature & 825 & $46.24 \%$ \\
Cold & 591 & $33.13 \%$ \\
Flat & 344 & $19.28 \%$ \\
Cool & 18 & $1.01 \%$ \\
Heat & 6 & $0.34 \%$ \\
\hline
\end{tabular}

Table 4. Analysis of five flavors of 80 prescription drugs

\begin{tabular}{ccc}
\hline Five flavors & Frequency & Frequency \\
\hline Bitter & 938 & $32.75 \%$ \\
Sweet & 825 & $28.81 \%$ \\
Symplectic & 770 & $26.89 \%$ \\
Acid & 176 & $6.15 \%$ \\
Light & 62 & $2.16 \%$ \\
Salty & 49 & $1.71 \%$ \\
Astringent & 44 & $1.54 \%$ \\
\hline
\end{tabular}




\subsection{Analysis of Drug Combination and Medication Association Rules}

SPSS modeler19.0 analysis software was used to analyze the association rules of major drugs with frequency greater than 23, and the minimum confidence was $80 \% .13$ groups of drug association rules were obtained (see Table 5). The drug association network diagram is shown in Figure 1. The thicker the line is, the stronger the association is.

Table 5. Analysis of correlation law of 80 prescription drugs (confidence $>0.8$ )

\begin{tabular}{cccc}
\hline consequent & The aforesaid & Support \% & Confidence $\%$ \\
\hline Curcuma & Wintersweet Flower & 35.00 & 100 \\
Rhaponticum uniflorum & Hive & 28.75 & 95.652 \\
Rhaponticum uniflorum & Bergamot & 35.00 & 92.857 \\
Rhaponticum uniflorum & Chinese atractylodes & 50.00 & 92.50 \\
Whitemustard seed & Corydalis yanhusuo & 55.00 & 86.364 \\
Whitemustard seed & Trichosanthes kirilowii & 53.75 & 86.047 \\
Atractylodes macrocephala Koidz & Wintersweet Flower & 35.00 & 85.714 \\
Rhaponticum uniflorum & Poria cocos & 38.75 & 83.871 \\
Paris polyphylla & Hive & 28.75 & 82.609 \\
Whitemustard seed & Wintersweet Flower & 35.00 & 82.143 \\
Rhaponticum uniflorum & Whitemustard seed & 70.00 & 82.143 \\
Rhaponticum uniflorum & Paris polyphylla & 70.00 & 82.357 \\
Paris polyphylla & Chinese atractylodes & 50.00 & 80.00
\end{tabular}

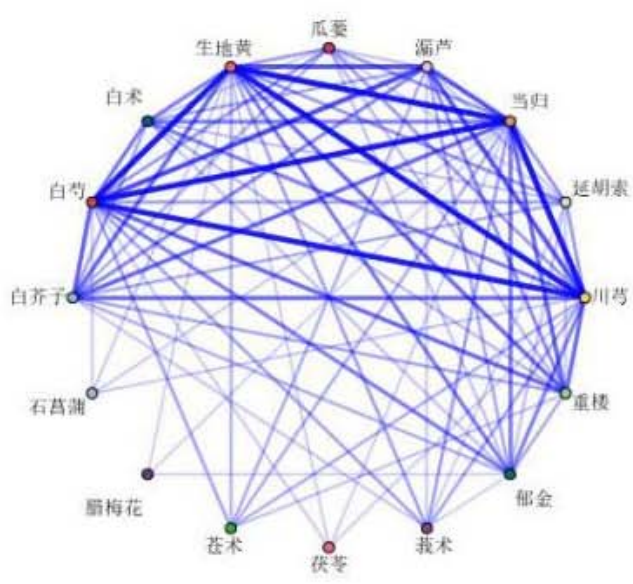

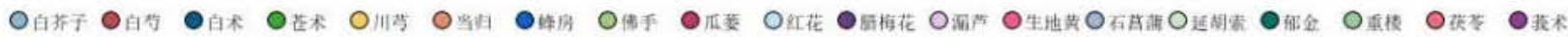

Figure 1. Network diagram of drug association rules.

\subsection{Cluster Analysis}

SPSS 19.0 software was used for cluster analysis of drugs with frequency more than 23, and then icicle diagram and tree diagram were drawn. As shown in Figure 2, if the cluster number is 10, four clusters can be obtained, of which three are effective clusters, namely: "safflower Curcuma", "Chimonanthus praecox Curcuma", "Trichosanthes kirilowii yanhusuo BAIJIEZI"; According to the tree diagram, 20 traditional Chinese medicines can be divided into 4 groups according to the cluster number of 15 points, among which the effective cluster groups are "Astragalus Radix Paeoniae Alba Loulu Chonglou", "BAIJIEZI yanhusuo" and "Yujin Chimonanthus", as shown in Figure 3. 


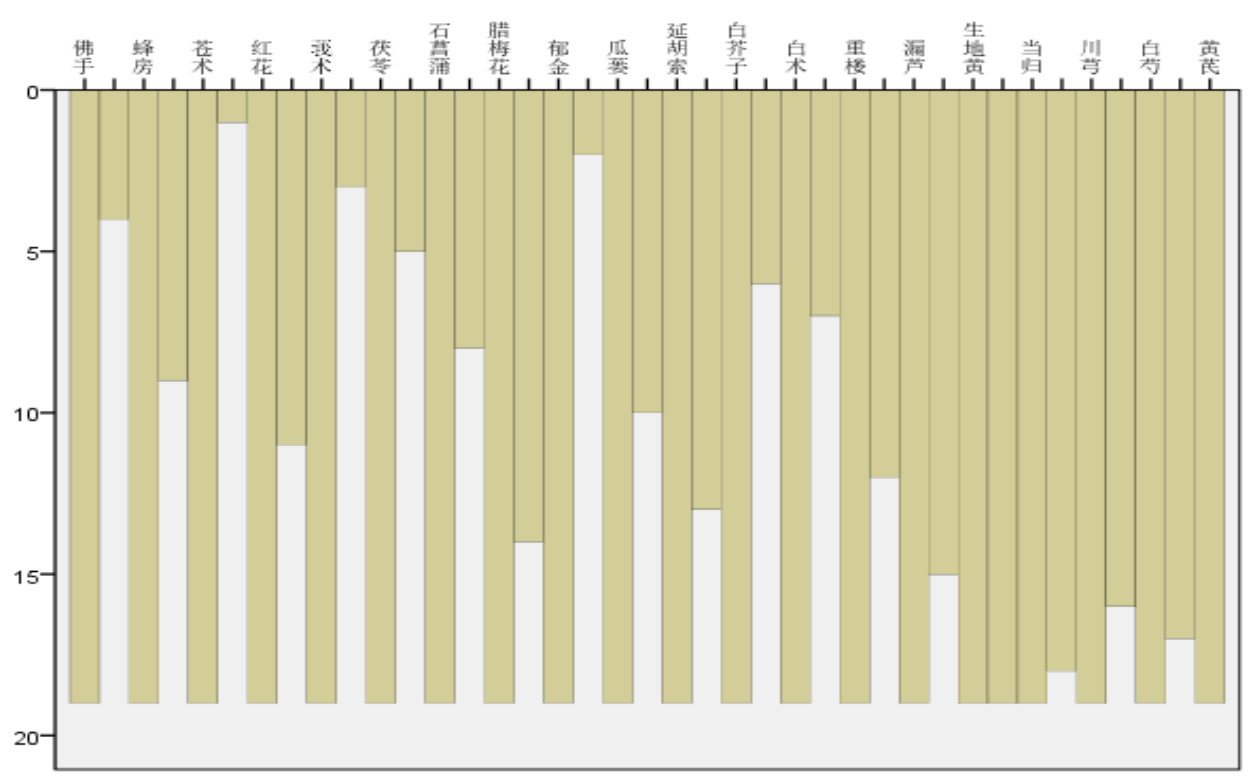

Figure 2. Icicle diagram of TCM treatment of hyperplasia of mammary glands.

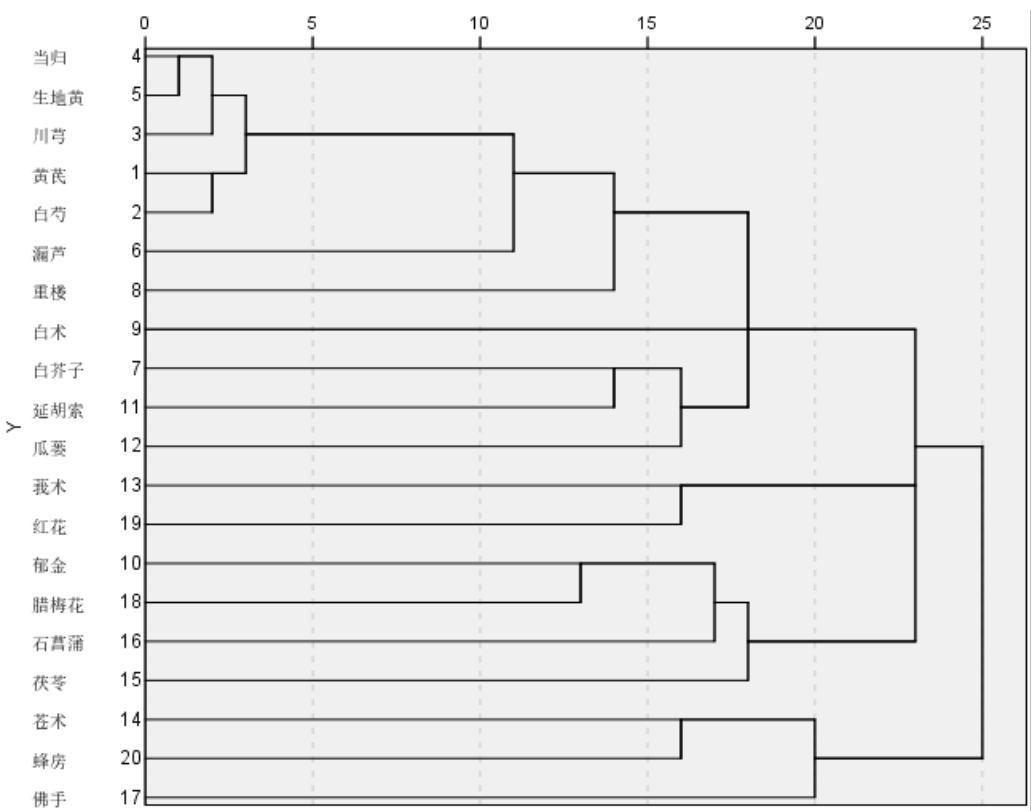

Figure 3. Dendrogram of high frequency main drugs in the treatment of hyperplasia of mammary glands with traditional Chinese Medicine.

Table 6. New prescriptions and combinations of cluster analysis

\begin{tabular}{cc}
\hline No. & New combination \\
\hline C1 & Radix astragali, Radix Paeoniae Alba, Rhizoma rhaponticum, Rhizoma Paridis \\
C2 & White mustard, corydalis \\
C3 & Curcuma and Chimonanthus praecox \\
\hline
\end{tabular}

\section{Discussion}

"Breast hyperplasia" is the name of modern medical disease, neither tumor nor inflammation. It is analyzed from the histological manifestations: it is hyperplasia and degenerative change of breast tissue. Modern medical research shows that most of the disease is closely related to endocrine dysfunction, bad living habits, psychological and mental factors. The above factors can cause imbalance of estrogen ratio and abnormal quality of sex hormone 
receptor, and lead to excessive or incomplete hyperplasia of breast substance and different degree of hyperplasia of various parts of breast, which leads to abnormal shape and quantity of breast structure [8-10]. Therefore, the effective regulation of estrogen and progesterone level and the improvement of endocrine function can treat breast hyperplasia [11].

Hyperplasia of mammary glands is called "mammophilia" in traditional Chinese medicine Yangke xindeji said: "There is tuberculosis in the breast, which is like a pill egg. It has no pain, no fever, and the skin color remains unchanged. Its nucleus changes with joy and anger. This is called mastia” [12]. The etiology of breast diseases was first recorded in the treatise on the origin of the princes, breast tuberculosis syndrome: "The meridians of Foot Yangming are from the lack of milk in the basin. The meridians are deficient, the wind is cold, the cold is broken in the blood, the swelling is formed... The cold cannot be eliminated, and the heavy fatigue is born". It is said in Surgery Orthodox [13] that "Ru Pi is tuberculosis in the breast, which is shaped like a pill egg. It is painful or not when it falls heavily, and its skin color remains unchanged. Its nucleus grows and wanes with joy and anger. It is mostly formed by thinking and hurting the spleen, irritating and hurting the liver, and stasis". It can be seen that mammary gland hyperplasia is mainly caused by poor emotion and excessive thinking, which leads to liver failure, qi stagnation, disharmony between liver and spleen or qi stagnation and blood stasis. Professor Yin kejing thinks that Chong Ren disorder is the main cause of mastia, liver depression and qi stagnation is the key to the formation, qi stagnation, blood stasis and phlegm coagulation is the "sign" of mastia.

In this study, a total of 80 prescriptions of Professor Yin Kejing for the treatment of hyperplasia of mammary glands were included, involving 170 kinds of traditional Chinese medicine. Among them, Astragalus membranaceus, Radix Paeoniae Alba, Ligusticum chuanxiong, Angelica sinensis and Rehmannia glutinosa were the top five herbs in the frequency of use; The meridian tropism of prescription drugs is mainly liver, spleen and heart; The four Qi of prescription drugs are mainly warm, cold, flat and cool, and the five flavors are mainly bitter, sweet and pungent. Professor Yin Kejing thinks that Qi and blood have two meanings. Astragalus membranaceus has the function of Invigorating Qi and Promoting Yang. It is an important medicine for Invigorating Qi. When it is matched with angelica, it can invigorate Qi and generate blood; in ancient times, Radix Paeoniae Alba, Rhizoma Chuanxiong, Radix Angelicae Sinensis and Radix Rehmanniae are the components of Siwu Decoction. Radix Angelicae sinensis, Radix Paeoniae Alba and Radix Rehmanniae have thick taste, which means Yin in Yin, so they can produce blood. Rhizoma Chuanxiong has thin taste and clear Qi, which means Yang in Yin, so they can promote qi in blood. The main channel of prescription drugs for the treatment of mastitis of yin and Lao were liver, spleen and heart; in the Qing Dynasty, ye Tianshi’s “clinical guide medical records” proposed that "Women are born with liver" [14]. The liver governs catharsis, regulates Qi in the whole body, stores blood and regulates blood volume; spleen is the foundation of postnatal, which is mainly used to transport and transform the essence of water and grain, so as to transform into blood; the heart governs the blood vessels, so that the blood runs continuously in the veins and circulates all over the body. Therefore, Professor Yin Kejing paid attention to regulating the liver, strengthening the spleen and tonifying the heart in the treatment of mastia. The drugs for promoting qi and activating blood circulation are mostly warm, the drugs for clearing heat and resolving stagnation are mostly cold, and the drugs for supplementing qi and nourishing blood are mostly flat; Yin Lao used both cold and warm medicine, which reflected the idea of tonifying and reducing. Bitter: "can discharge, can be dry, can be strong”, with Ruanjian Sanjie, dampness resolving phlegm, purging fire and Yin and other effects, modern pharmacological research confirmed that Ruanjian Sanjie drugs can help stimulate the secretion of luteinizing hormone, can improve luteal function [15], and can inhibit the replication function of proliferative cells, make the proliferative breast tissue return to normal [16]; Gan: "can be tonic, can be harmonious, can be slow", has the function of tonifying vital energy, relieving pain, tonifying qi and nourishing blood, etc; Xin: "can do, can disperse”, has the function of divergence, Qi, blood and so on. Professor Yin kejing mainly used "tonifying blood with sweetness, invigorating blood with bitterness, softening firmness and dispersing knot with bitterness".

In this study, according to the results of drug association analysis support and drug association rules network diagram, the most commonly used drug combinations in clinic are "Yujin Chimonanthus", "Loulu beehive", "Loulu Bergamot". Yujin is cold in nature, bitter and pungent in taste. It belongs to the liver, gallbladder and heart meridians. It is the Qi medicine in the blood. "Practical Chinese medicine and prescription" says: "Yujin can soothe the liver and dredge the lung”. It is good at promoting blood circulation to relieve pain, promoting qi to relieve depression, and is good at treating liver depression, qi stagnation and blood stasis. Modern pharmacological research shows that Yujin has the pharmacological effects of hemostasis, anticoagulation, vasoconstriction, anti inflammation and analgesia, negative muscle strength, anti-oxidation and liver protection [17]; the flower of Chimonanthus praecox is warm in nature and pungent in taste. It belongs to liver, stomach and lung meridians. It has the functions 
of clearing away heat and toxin, regulating qi and relieving depression. Pharmacological studies have shown that Chimonanthus praecox has the functions of anti-inflammatory and antibacterial, analgesic and antitussive, antiviral, and scavenging free radicals [18]. Professor Yin Kejing thinks that Chimonanthus praecox can regulate qi and relieve depression, and Yujin can soothe liver and relieve depression. The two drugs complement each other and play the role of soothing liver and relieving depression, promoting blood circulation and relieving pain. It is cold in nature and bitter in taste. It belongs to the stomach meridian. It has the functions of clearing away heat and toxin, removing carbuncle, removing milk, relaxing tendons and activating pulse. Modern pharmacological studies show that it has the functions of enhancing immunity, anti inflammation and analgesia [19]. It has the functions of attacking poison, killing insects, dispelling wind and relieving pain; Yin Lao believed that the combination of Loulu and beehive could clear away heat, detoxify, detumescence and resolve knots, and had a significant therapeutic effect on mastia. Bergamot is warm, pungent, bitter and sour. It belongs to liver, spleen, stomach and lung meridians. It has the functions of soothing liver and regulating qi, harmonizing stomach and relieving pain, drying dampness and resolving phlegm. Yin Lao believed that bergamot and Liulu could be used to soothe liver and regulating qi, eliminate carbuncle and disperse stagnation. According to the network diagram of drug association rules, Angelica sinensis, chuanxiong, Paeoniae Alba and Rehmannia glutinosa are most closely related. Professor Yin Kejing thinks that the treatment of mastia should start with Qi and blood, which is the foundation of women. Angelica sinensis can replenish blood and activate blood, Chuanxiong can activate blood and activate Qi, Paeoniae Alba can nourish blood and soften liver, and Rehmannia can clear heat and cool blood. It is good to bleed without hurting.

According to the cluster analysis of the top 20 traditional Chinese medicines in the prescription, it is found that there are three effective clusters in the icicle diagram according to the cluster number of 10, which are: Safflower zedoary turmeric, Chimonanthus praecox Curcuma, Trichosanthes kirilowii yanhusuo BAIJIEZI; Professor Yin Kejing thinks that the main reason of hyperplasia of mammary glands is the imbalance of Chong and Ren, which makes Qi and blood stagnate. Safflower has the function of dispersing blood stasis and relieving pain, activating blood circulation and unblocking meridians. Curcuma has the function of breaking blood circulation and promoting Qi, eliminating accumulation and relieving pain. They are compatible with each other, which can promote blood circulation and relieve pain, disperse blood stasis and promote qi, and are good at treating the pain of blood stasis, Inhibit the synthesis of collagen fibers, so as to promote the disappearance of proliferative masses [15]; Professor Yin Kejing thinks that the key to the formation of hyperplasia of mammary glands is stagnation of liver qi, unsmooth emotion, long depression and liver injury. Chimonanthus praecox has the functions of clearing away heat and toxin, regulating qi and relieving depression. Yujin has the functions of promoting blood circulation and relieving pain, promoting qi and relieving depression. The two drugs complement each other to achieve the effects of soothing liver, relieving depression and relieving pain; Professor Yin Kejing believes that the "standard" of mammary gland hyperplasia is Qi stagnation, blood stasis and phlegm coagulation, Qi is the first of six depression, qi depression is located in the liver, liver depression by spleen, affecting the function of spleen and stomach transportation, transportation and transformation dereliction of duty, body fluid is not distributed, blood stasis does not disperse, dampness depression is combined, blood stasis and phlegm coagulation, phlegm turbidity and blood stasis, accumulation of milk collaterals, is the standard of mammary gland hyperplasia, Yanhu rope has the functions of promoting Qi, activating blood circulation and relieving pain. BAIJIEZI has the functions of warming lung, eliminating phlegm and promoting Qi, dispersing collaterals and relieving pain. The combination of the three can achieve the functions of promoting qi and relieving pain, detumescence and dispersing knots. Professor Yin Kejing thinks that there are many pathogenesis of hyperplasia of mammary glands, the main pathogenesis is the imbalance of Chong and Ren, stagnation of liver qi and stagnation of Qi, blood stasis and phlegm coagulation. According to the cluster analysis of the top 20 traditional Chinese medicines in the prescription, three new prescription combinations can be obtained from the tree diagram. Among the C1 combinations, Astragalus membranaceus, Radix Paeoniae Alba, Rhizoma rhaponticum and Rhizoma Paridis. The combination of Astragalus membranaceus and Radix Paeoniae Alba can replenish qi, nourish blood and soften the liver. The combination of Rhizoma Paridis and Rhizoma rhaponticum can clear away heat and toxin, and eliminate carbuncle. Professor Yin thinks that the four drug combinations can be used as new candidate prescriptions for the treatment of Chong Ren syndrome, Combined use of all kinds of medicine, treating both symptoms and signs, tonifying qi and blood, promoting qi and removing blood stasis [20]; In C2 combination, there are white mustard seed and corydalis. They can activate Qi and blood circulation, disperse knot and relieve pain. They have good effect in the treatment of hyperplasia of mammary glands with qi stagnation, blood stasis and phlegm coagulation type; in combination C3, there are Curcuma and Chimonanthus praecox. The combination of the two drugs has the effects of promoting blood circulation, relieving 
pain, soothing liver and relieving depression. It has a significant effect on the treatment of hyperplasia of mammary glands with liver qi stagnation.

Based on data mining, this study analyzed Professor Yin Kejing's prescription for the treatment of mammary gland hyperplasia, preliminarily summarized and summarized its medication rules and medication characteristics, obtained some traditional Chinese medicine experience, summarized the new knowledge and new information, and provided reference for Professor Yin Kejing's clinical experience and inheritance in the treatment of mammary gland hyperplasia. Combined with Professor Yin Kejing's clinical diagnosis and treatment experience, the results of this study are objective and true, which provides a certain reference for the inheritance of the experience of famous old Chinese medicine, and provides a basis for the clinical treatment of breast hyperplasia.

\section{References}

[1] Ma Wei, Jin Quanxiu, Wu Yunfei, Jin Feng. (2016). Expert consensus on diagnosis and treatment of breast hyperplasia [J]. Chinese Journal of Practical Surgery, 2016, 36(07): 759-762.

[2] Wu Yue, Wu Yongqiang, Gao Xiufei, Wang Bei, Zheng Hongbin, Zhang Shuo. (2016). Clinical effect of Chaihu Shugan San and Ganmai Dazao Decoction on the treatment of breast hyperplasia [J]. Journal of Zhejiang University of Traditional Chinese Medicine, 2016, 40(06): 434-438.

[3] Li Yueqing. (2008). Chinese medicine surgery. Beijing: China traditional Chinese Medicine Press, 2008: 113.

[4] Liu Deguo, Li Ziliang, Liu Kai, Chen Xingyuan, Fangying, Fan Hongqiao, Hu Jinhui. (2016). Data mining of the law of drug use in ancient and modern breast cancer (mastoid) [J]. Journal of Hunan University of Traditional Chinese Medicine, 2016, 36(04): 70-73.

[5] Jiang Jun. (2008). Hyperplasia of breast: clinical problems worthy of attention [J]. Chinese Journal of Breast Disease (Electronic Edition), 2008(01): 13-19.

[6] Gao Xuemin Chinese Medicine (m). 2 ed. Beijing: China traditional Chinese Medicine Press, 2010.

[7] Liu Yongxin. (2011). National pharmacopoeia practical manual of traditional Chinese medicine [M]. Beijing: Ancient Chinese Medicine Press, 2011.

[8] Lin Yi, Tang hanjun. (2003). Breast disease of modern Chinese medicine [M]. Beijing: People’s Health Press, 2003.

[9] Jiang Wenting, Zhang Min. (2016). Xiaopi San Jie Decoction and emotional therapy of traditional Chinese medicine in the treatment of breast hyperplasia [J]. Journal of Traditional Chinese Medicine, 2016, 31(12): 1995-1998.

[10] Zheng Shenghong. (2018). Wang Youren's experience in treating hyperplasia of mammary glands with tongtiaoyin method [J]. Chinese Journal of Traditional Chinese Medicine Information, 2018, 25(06): 115-117.

[11] Yang Yulong. (2018). Clinical study on 45 cases of hyperplasia of mammary glands treated with Xiaojie Decoction and Shuru powder for external use [J]. Asia Pacific Traditional Medicine, 2018, 14(04): 175-176.

[12] Wang Shuxin, Duan Biao, Chen Yujing, Liu Huanling, He Xueping, Chen Hanwei. (2017). Study on the medication regularity of traditional Chinese medicine in the treatment of breast hyperplasia [J]. Chinese Medicine Guide, 2017, 23(23): 50-53.

[13] Wu Yue, Chen Ruiping. (2018). Professor Ruan Guozhi’s experience in treating hyperplasia of mammary glands [J]. Research of Traditional Chinese Medicine, 2018, 31(03): 46-48.

[14] Wen Yinjun. (2019). Research on the academic thought and clinical experience of Ye Tianshi in treating gynecological diseases [D]. Anhui University of traditional Chinese medicine, 2019.

[15] Guo Yue. (2011). Clinical observation on the treatment of hyperplasia of mammary glands with integrated traditional Chinese and Western Medicine [J]. Chinese Community Physician (Medical Specialty), 2011, 13(17): 189-190.

[16] Peng Huaiqing. (2014). Yiqi Xiaohe decoction combined with BAIJIEZI ointment for moxibustion in the treatment of 62 cases of hyperplasia of mammary glands [J]. National Medical Forum, 2014, 29(02): 28-29.

[17] Yu Xianwen, Chen Qiongfang, Wang Gang, Yang Xiufen. (2017). Research progress on chemical constituents and pharmacological effects of Curcuma Kwai [J]. Chinese Journal of Traditional Chinese Medicine, 2017, 35(07): 1891-1893.

[18] Du Yufang, Chen Haifang, Feng Yulin, Song Yonggui, Zhang Wugang, Yang Jie, Yang Shilin. (2013). Research progress on chemical constituents and pharmacological effects of Chimonanthus [J]. Shi Zhen, Guoyi Guoyao, 2013, 24(08): $1981-1983$.

[19] Yang Meizhen, Wang Xiaoqin, Liu Yong, Du Yue. (2015). Studies on chemical constituents and pharmacological activities of rhaponticum uniflorum [J]. Chinese Patent Medicine, 2015, 37(03): 611-618.

[20] Du Yiming, Du Xu, Chu Wangchong, Yin Kejing. (2020). Clinical observation on treating blood stasis and phlegm coagulation type of hyperplasia of mammary glands with acupuncture and medicine [J]. Journal of Liaoning University of Traditional Chinese Medicine, 2020, 22(01): 174-177. 